\section{SOI: 1.1/TAS DOI: 10.15863/TAS International Scientific Journal Theoretical \& Applied Science}

p-ISSN: 2308-4944 (print) e-ISSN: 2409-0085 (online)

Year: $2015 \quad$ Issue: 05 Volume: 25

Published: 30.05.2015 http://T-Science.org

SECTION 4. Computer science, computer engineering and automation.
Nikita Sergeevich Drozdovsky

master of

North-Caucasus Federal University, Stavropol, Russia nikita_drozdovsk@mail.ru

Pavel Vladimirovich Dvoryaninov

master of

North-Caucasus Federal University, Stavropol, Russia paveldv92@mail.ru

Yaroslav Yurievich Lenskiy master of

North-Caucasus Federal University, Stavropol, Russia yalenskiy@yandex.ru

\title{
REALIZATION OF ALGORITHMS ON COLUMNS AND TREES
}

Abstract: Many tasks can be solved by means of one of the most powerful mathematical tools - counts. And if defined that this task - a task on columns, it can already be considered a half of the carried-out task. But solving such problems if data can be presented not only in the form counts but also as trees, then it is possible to construct very effective decision. It is possible to present any structure or system in the form of counts, from system of transportation to a data transmission network. They can be even more useful if to add to them additional data like scales or distances, it gives the chance to describe such difficulties as forecasting of game in chess or definition of suitable work for the person proceeding from his opportunities. Trees - represent a special type of counts so the most part of algorithms and representations of counts work and for them. Due to their special parameters, it is possible to apply special versions of algorithms and representations. In practice there are cases when structures which need to be presented in the form of trees meet. Thus these such cases pretty general.

Key words: Python, graphs, trees.

Language: Russian

Citation: Drozdovsky NS, Dvoryaninov PV, Lenskiy YY (2015) REALIZATION OF ALGORITHMS ON COLUMNS AND TREES. ISJ Theoretical \& Applied Science 05 (25): 62-68.

Soi: http://s-o-i.org/1.1/TAS*05(25)13 Doi: crossef http://dx.doi.org/10.15863/TAS.2015.05.25.13

\section{РЕАЛИЗАЦИЯ АЛГОРИТМОВ НА ГРАФАХ И ДЕРЕВЬЯХ}

Аннотация: Многие задачи могут быть решены при помощи одного из мощнейших математических инструментов - графов. И если определили, что данная задача - задача на графы, то это уже можно считать половиной выполненной задачи. Но решая такие задачи, если данные можно представить не только в виде графов, но и как деревья, тогда можно построчть очень эффективное решение. $B$ виде графов можно представить всякую структуру или систему, от системь транспортировки до сети передачи данных. Они могут быть еще более полезньми, если добавить в них дополнительные данные вроде весов или расстояний, это дает возможность описывать такие трудности как прогнозирование игры в шахматы или определение подходящей работы для человека исходя из его возможностей. Деревья представляют особый вид графов, так что большая часть алгоритмов и представлений графов работают и для них. В связи с их особенными параметрами, можно применять особые версии алгоритмов и представлений. На практике же бывают случаи, когда встречаются структуры, которые необходимо представить в виде деревьев. При это эти такие случаи довольно-таки общие.

Ключевые слова: Python, графы, деревья.

Описание задачи в терминах графов является достаточно абстрактным, поэтому, когда реализуется решение, нужно представить графы в виде определенной структуры данных. Структура данных не потребуется только в тех случаях, если граф будет уже составлен в коде или данных. Имеется метод реализации функции смежности, $\mathrm{N}(\mathrm{v})$, так, чтобы $\mathrm{N}[\mathrm{v}]$ было любым набором смежных с v вершин.
Хэширование - это вычисление целого числа, основанного на произвольном объекте, которое можно использовать, к примеру как индекс в массиве. Эта техника, неявно используется программистами на Python $<\mathrm{em}>$ хэширование $</ \mathrm{em}>$.

Механизм хэширования в Python представлен функцией hash:

>> hash("TEXT") 
В словарях этот механизм употребляется как хэширование, реализованных как так называемые хэш-таблицы. Большинство из них реализованы этим же методом. Его главная особенность заключается в том, что хэш может быть вычислен по существу за константное время, если существует довольно большой массив, то доступ к нему при помощи хэша в среднем занимает $\Theta(1)$. Это значит, что доступ к элементам в dict или set происходит за постоянное время. Именно это и делает их очень полезными строительными блоками для более сложных структур и алгоритмов.

Списки смежности являются одним из наиболее распространенных и обычных методов представления графов. Их суть заключается в том, что для каждой вершины создается список смежных с ней вершин. Ниже рассмотрим простой метод реализации списка смежности.

Предположим, что имеется $\mathrm{n}$ вершин, пронумерованных $0 \ldots$ n-1. Эти вершины могут быть представлены любыми объектами, иметь свои метки и имена. Использование целых чисел в диапазоне $0 \ldots \mathrm{n}-1$ делает реализацию большинства представлений намного проще. Именно в таком случае номера вершин можно использовать как индексы. Потому каждый список смежности является списком таких номеров. Списки же собраны в один главный список размера n, индексированный номерами вершин. В большинстве случаев такая сортировка списков случайная, поэтому эти списки можно использовать для реализации множеств смежностей. В Рython имеется отдельный тип для множеств. Этот тип в большинстве случаев удобно использовать.

Примерный граф разных видов представлений изображен на рисунке 1 . Предположим, что все вершины пронумерованы $(\mathrm{a}=0, \mathrm{~b}=1, \ldots)$. Как можно представить граф простым способом показано в листинге ниже. Для удобства использования надо присвоить номера вершин переменным, названным в соответствии с метками вершин на рисунке 1 . С числами можно работать так же напрямую. Какой вершине, какой список принадлежит указанно в комментариях.

Листинг 1.

a,b,c,d,e,f,g, h = range( 8 )

$\mathrm{N}=[$

$\{\mathrm{b}, \mathrm{c}, \mathrm{d}, \mathrm{e}, \mathrm{f}\}, \# \mathrm{a}$

$\{\mathrm{c}, \mathrm{e}\}, \# \mathrm{~b}$

$\{\mathrm{d}\}, \# \mathrm{c}$

$\{\mathrm{e}\}, \# \mathrm{~d}$

$\{\mathrm{f}\}$,\#e

$\{\mathrm{c}, \mathrm{g}, \mathrm{h}\}, \# \mathrm{f}$

$\{\mathrm{f}, \mathrm{h}\}, \# \mathrm{~g}$

$\{\mathrm{f}, \mathrm{g}\} \# \mathrm{~h}]$

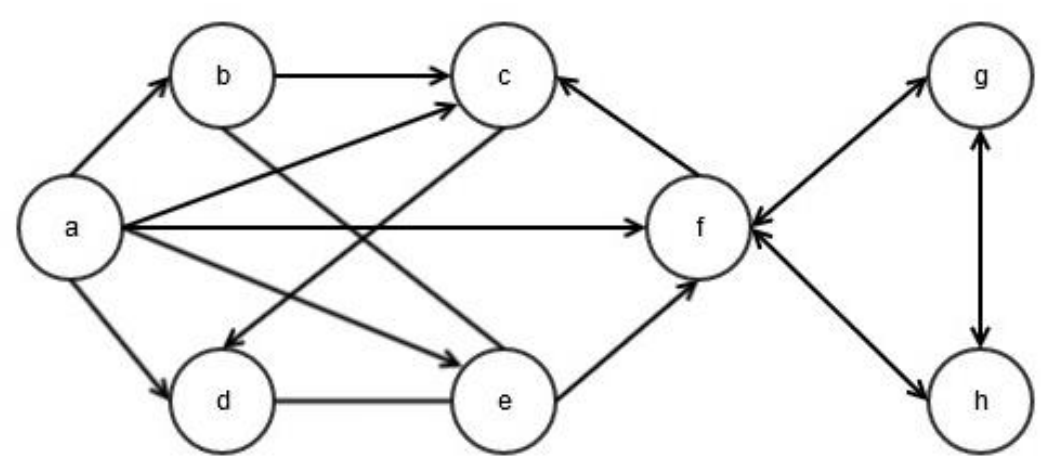

Рисунок 1 - Граф разных видов представления.

Имя $\mathrm{N}$ из листинга 1 связано с функцией $\mathrm{N}$, описанной выше. В теории графов $\mathrm{N}(\mathrm{v})$ представляет множество смежных c v вершин. Так же и в коде $\mathrm{N}[\mathrm{v}]$ является множеством смежных с $\mathrm{v}$ вершин. Предполагая, что $\mathrm{N}$ определена как в примере выше, в интерактивном режиме Python выглядит это представление:

\section{$\gg$ b in $\mathrm{N}[\mathrm{a}]$ \# смежная? \\ True \\ >> len(N[f]) \# степень 3}

Если у есть определенный код в файле и необходимо запустить этот код в интерактивном режиме, то нужно запустить python c ключом -i, например так: python -i listing_2_1.py

Данная команда запустит код из файла и откроет интерактивный интерпретатор. Он продолжит работать в месте, где закончился код в файле и сохранит все сделанные раньше объявления.

Списки смежности являются другим возможным представлением. В определенных случаях он даст меньше накладных расходов. Пример такого списка приведен в листинге ниже. В этих списках доступны все те же операции, но при этом проверка смежности вершины выполняется за $\Theta(n)$, что дает серьезное снижение скорости. 


\section{Листинг 2}

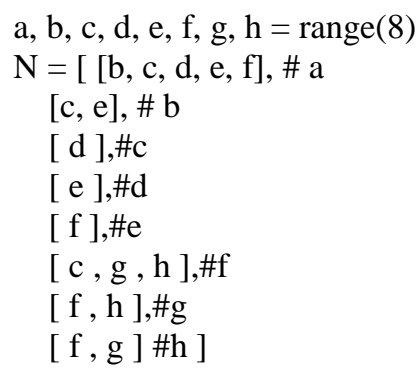

Это представление все же является традиционным списком смежности, а не простым набором массивов, поскольку тип «список» в Python является динамическим массивом. Вместо типа list из Python же можно реализовать тип связанного перечня. Делается это для дешевизны в плане производительности произвольных вставок в списки. Но при этому главное преимущество использования встроенного list заключается в том, что он представляет собой очень быструю и хорошо отлаженную структуру.

Работая с графами можно понять, что самое лучшее представление будет находится в зависимости от того, что именно необходимо сделать с графом. Если использовать списки смежности, то можно сохранить затратные расходы небольшими и обеспечить эффективный обход $\mathrm{N}(\mathrm{v})$ для любой вершины v. Но проверка, являются ли u и v смежными, потребует времени $\Theta(\mathrm{N}(\mathrm{v}))$. Это станет большой проблемой при высокой плотности графа. В такой ситуации лучше использовать множества смежности. Можно удалить объекты из середины list в Python, но это достаточно затратно. При этом удаление с конца происходит за константное время. За константное время можно удалить случайную вершину и перезаписать ее той, которая находиться в конце списка смежности. При это вызывая метод рор, если не нужно заботиться о порядке вершин.

Сортированные списки смежных вершин являются некой вариацией на тему этого представления. Если списки не очень часто изменяются и их можно держать постоянно отсортированными, то для проверки смежности вершин можно использовать бисекцию. Единственный минус заключается в том, что это увеличит сложность проверки до $\Theta(\log 2 \mathrm{k})$, где k - количество смежных с данной вершин. Но при этом это приведет к меньшим затратным расходам, в плане использования памяти и времени итерации.

Так же небольшой доработкой является замена множеств или списков на словари. Смежные вершины - являются ключам словаря, а в качестве значения можно использовать любые дополнительные данные, например, вес ребра. Как это выглядит можно увидеть в листинге ниже.
Листинг 3.

$\mathrm{a}, \mathrm{b}, \mathrm{c}, \mathrm{d}, \mathrm{e}, \mathrm{f}, \mathrm{g}, \mathrm{h}=\operatorname{range}(8)$

$\mathrm{N}=[\{\mathrm{b}: 2, \mathrm{c}: 1, \mathrm{~d}: 3, \mathrm{e}: 9, \mathrm{f}: 4\}, \# \mathrm{a}$

$\{\mathrm{c}: 4, \mathrm{e}: 3\}, \# \mathrm{~b}$

$\{\mathrm{d}: 8\}$, \# c

$\{\mathrm{e}: 7\}, \# \mathrm{~d}$

$\{f: 5\}, \#$ e

$\{\mathrm{c}: 2, \mathrm{~g}: 2, \mathrm{~h}: 2\}, \# \mathrm{f}$

$\{\mathrm{f}: 1, \mathrm{~h}: 6\}, \# \mathrm{~g}$

$\{\mathrm{f}: 9, \mathrm{~g}: 8\}$ \# h ]

Словарь смежности можно использовать точно так же, как и другие представления, с учетом дополнительной информации о весах:

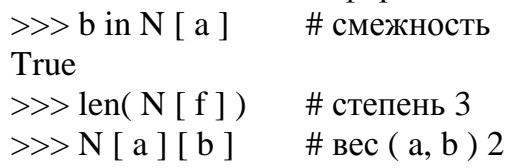

Так же можно использовать словари смежности даже если нет полезных данных вроде весов ребер - это даст преимущества множеств смежности, но при этом будет работать со старыми версиями Python, не имеющими поддержки типа set.

До этого момента сущность, хранящая структуры

смежности являлась списком, множества или словари - список, индексированный номерами вершин. На основе словаря можно построить более гибкий вариант основной структуры. Ниже приведен пример словаря, содержащего множества смежностей, вершины в нем обозначены символами.

Листинг 4.

$\mathrm{N}=\{$ 'a': set( 'bcdef' $)$,

'b': set( 'ce' ),

'c': set( 'd' ),

'd': set( 'e' ),

'e': $\operatorname{set}($ ' $f$ ' ),

'f': set( 'cgh' ),

'g': set( 'fh' ),

'h': set( 'fg' ) \}

В данном листинге без конструктора set останутся только строки смежности, которые будут работать как списки смежности из символов.

Матрица смежности - так же одна из популярных форма представления графов. Основное их отличие заключается в следующем: вместо перечисления всех смежных с каждой из вершин, записывается один ряд значений, каждое из которых соответствует возможной смежной вершине. И сохраняет значение, показывающее, действительно ли вершина является смежной. И вновь простейшую реализацию можно получить используя вложенные списки, это также требует, чтобы вершины были пронумерованы от 0 до V1. В качестве значений истинности используются 1 и 0 вместо False и True, чтобы сделать матрицу читабельной. 
Листинг 5.

$$
\begin{aligned}
& \text { a, b, c, d, e, f, g, h }=\operatorname{range}(8) \\
& \# \text { a b c d e f g h } \\
& \mathrm{N}=[[0,1,1,1,1,1,0,0], \# \mathrm{a} \\
& {[0,0,1,0,1,0,0,0], \# \mathrm{~b}} \\
& {[0,0,0,1,0,0,0,0], \# \mathrm{c}} \\
& {[0,0,0,0,1,0,0,0], \# \mathrm{~d}} \\
& {[0,0,0,0,0,1,0,0], \# \text { e }} \\
& {[0,0,1,0,0,0,1,1], \# \mathrm{f}} \\
& {[0,0,0,0,0,1,0,1], \# \mathrm{~g}} \\
& [0,0,0,0,0,1,1,0]] \# \mathrm{~h}
\end{aligned}
$$

Метод использования матриц смежности немного отличается от множеств и списков смежности. Вместо проверки входит ли b в $\mathrm{N}[\mathrm{a}]$, будет проверяться, истинно ли значение ячейки матрицы $\mathrm{N}[\mathrm{a}][\mathrm{b}]$. Кроме того, больше нельзя использовать len(N[a]), чтобы получить количество смежных вершин, потому что все ряды одной и той же длины. Вместо этого можно использовать функцию sum:

$$
\begin{aligned}
& \gg>\mathrm{N}[\mathrm{a}][\mathrm{b}] 1 \\
& \gg>\operatorname{sum}(\mathrm{N}[\mathrm{f}]) 3
\end{aligned}
$$

Матрицы смежности имеют ряд нужных параметров, о которых стоит знать. Во-1-х, потому что мы не рассматриваем графы с петлями, все значения на диагонали ложны. Также, ненаправленные графы обычно описываются парами ребер в обоих направлениях. Это означает, что матрица смежности для ненаправленного графа будет симметричной.

Расширение матрицы смежности для использования весов тривиально: вместо сохранения логических значений, сохраняйте значения весов. В случае с ребром (u, v) N[u][v] будет весом ребра w(u,v) вместо Тrue. Часто в практических целях несуществующим ребрам присваиваются бесконечные веса. Не всегда очевидно, как представить бесконечность, но совершенно точно есть несколько разных вариантов.

Один из вариантов заключается в том, что используется некорректный вес значения, такое как None или -1, если все веса являются неотрицательными. В некоторых случаях эффективнее использовать действительно большие числа. Если веса являются целые числа, то можно применить sys.maxint, хотя это значение не обязательно должно быть самым большим. Для отражения бесконечности существует значение inf. B Python напрямую по имени оно недоступно и выражается: float( 'int' ).

Листинг ниже показывает, как выглядит матрица весов, реализованная вложенными списками. Использованы те же веса, что и в листинге выше.

Листинг 6.

$\mathrm{a}, \mathrm{b}, \mathrm{c}, \mathrm{d}, \mathrm{e}, \mathrm{f}, \mathrm{g}, \mathrm{h}=\operatorname{range}(8)$

$$
\begin{aligned}
& =\text { float ('inf') } \\
& \text { \# a b c d e f g h } \\
& \mathrm{W}=\left[\left[0,2,1,3,9,4,,_{-}\right], \# \mathrm{a}\right. \\
& \text { [_, } 0,4,, 3,3,,,], \text { \# b } \\
& \text { [_,_, } \left.0,8,{ }_{-},{ }_{-},{ }_{-}\right], \text {\# c } \\
& \text { [_,_,_, } 0,7, \ldots,,,], \# \mathrm{~d} \\
& \text { [_,_,,_, } \left.0,5,{ }_{-},\right], \text {\# e } \\
& \text { [_,,2,_,_, 0,2,2], \# f } \\
& \text { [_,_,_,_,_, } 1,0,6], \# \mathrm{~g} \\
& \text { [_,_,_,_,_,9,8,0]] \# h }
\end{aligned}
$$

Бесконечное значение обозначено как подчеркивание ( $\_$), потому что это коротко и визуально различимо. Так же это означает что можно использовать абсолютно любое имя. Посмотрев на матрицу весов можно увидеть, что по диагонали, что даже без учета петель, все веса интерпретируются как расстояния, а расстояние от вершины до самой себя - равно нулю.

Конечно, матрицы весов дают возможность очень просто получить веса ребер, но, к примеру, проверка смежности и определение степени вершины, или обход всех смежных вершин делаются иначе. Здесь нужно использовать бесконечное значение, примерно так:

> W [ a ] [ b ] < inf \# смежность

True

>> W [ c ] [ e ] < inf \# смежность

False

$\gg \operatorname{sum}(1$ for $w$ in $\mathrm{W}[\mathrm{a}]$ if $w<$ inf $)-1 \#$ степень 5

Из полученной степени вычитается 1, так как не считаются значения на диагонали. Сложность вычисления степени здесь $\Theta(\mathrm{n})$, в то время как в другом представлении и смежность, и степень вершины можно найти за константное время. Так что всегда нужно понимать, как именно будет использоваться граф и выбирать для него соответствующее представление.

Библиотека NumPy содержит много функциональности, связанной с многомерными массивами. Для представления графов большая ее часть не нужна, но массивы из NumPу очень полезны, к примеру, для реализации матриц весов либо смежности.

Вместо создания пустой матрицы весов или смежности из списков для $\mathrm{n}$ вершин, вроде такого:

$\gg>\mathrm{N}=\left[[0]^{*} 10\right.$ for $\mathrm{i}$ in range $\left.(10)\right]$

в NumPy можно использовать функцию zeros:

>> import numpy as np

>> $\mathrm{N}=$ np.zeros $([10,10])$

Отдельные элементы доступны по индексам, разделенным запятой: $\mathrm{A}[\mathrm{u}, \mathrm{v}]$. Чтобы получить соседние с данной вершины, используется одиночный индекс: $\mathrm{A}[\mathrm{u}]$.

Необходима та версия NumPy, что будет работать с установленной версией Руthon. Если последний релиз NumPy не поддерживает ту версию Python, что используется, то можно 
скомпилировать и установить библиотеку прямо из репозитория исходных кодов. Исходный код можно получить при помощи следующих команд.

Реализация деревьев.

Деревья - особый вид графов. Абсолютно все представления графов можно использовать для представления деревьев. Представления деревьев играет большую роль, в отличии от простых графов, при использовании их в алгоритмах для них создано множество структур и методов. Множество алгоритмов на деревьях можно рассматривать в терминах теории графов и специально созданные структуры данных делают их реализацию гораздо проще. Самое простое представление, является представления дерева с корнем, в котором ребра спускаются вниз от корня. Это представление проще всего описать. В таких деревьях часто отображается иерархическое ветвление данных. Корень в низ показывает все объекты, а все внутренние узлы - указывают на объекты, содержащиеся в дереве. Корень каждого объекта - тот самый узел. Это описание можно использовать, представив каждое поддерево списком, содержащим все его поддеревья-потомки. Простое дерево с отмеченным путем от корня к листу, показано на рисунке 2.

Можно представить это дерево как список списков:

$$
\gg \mathrm{T}=\text { [ [ " a ", " b " ], [ " c " ], [ " d ", [ " e ", }
$$$$
\text { "f " ] ] ] }
$$

$\gg \mathrm{T}[0][1]$ 'b'

$\gg$ T [2] [ 1 ] [0] 'e'

Каждый список в сущности является списком потомков каждого из внутренних узлов. Во 2-м примере идет обращение к третьему потомку корня, после ко второму его потомку и в конце концов - к первому потомку предшествующего узла.

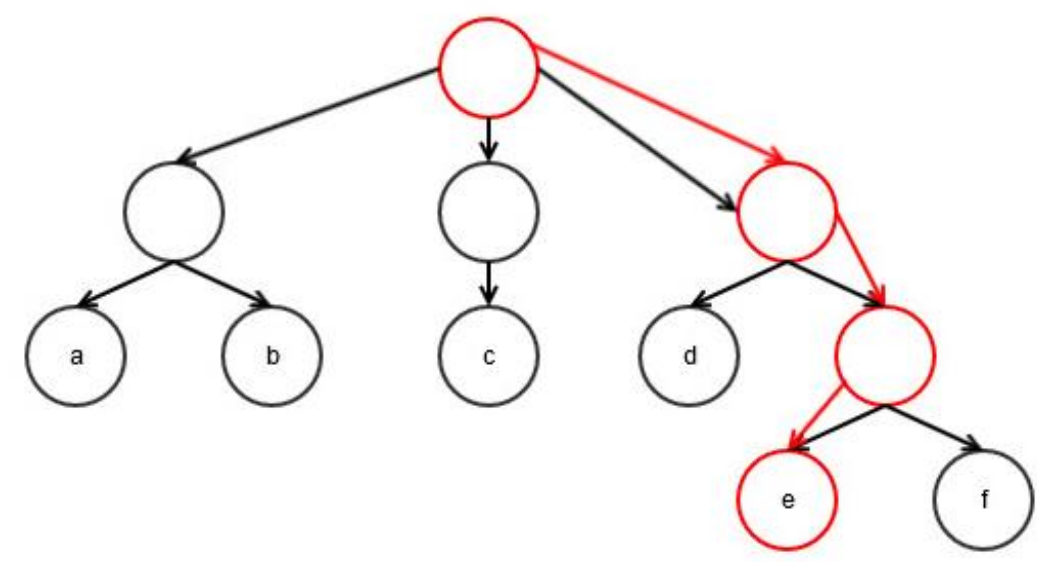

Рисунок 2 - Пример дерева с отмеченным путем от корня к листу.

В большинстве случаев можно заранее определить максимальное число потомков каждого узла. В связи с этим можно использовать объекты с отдельными атрибутами для каждого потомка как показано в листинге ниже.

Листинг 7.

class Tree:

$$
\begin{aligned}
& \text { def__init_( self, left, right ): } \\
& \text { self.left }=\text { left } \\
& \text { self.right }=\text { right } \\
& \text { >> t = Tree( Tree( " a ", " b " ), Tree( " c ", " d } \\
& \text { >> t.right.left }
\end{aligned}
$$$$
\text { ") ) }
$$$$
\text { 'c' }
$$

None можно использовать для того чтобы обозначить отсутствующих потомков. При этом комбинировать можно разные методы.

Один из самых распространенных методов для реализации деревьев, особенно для языков не имеющих встроенной поддержки списков, это представление - «Первый потомок, следующий брат». В этом методе каждый два указателя или атрибута. Которые указывают на другие узлы, так как это представляется в бинарном дереве. Первый из этих атрибутов ссылается на первого потомка узла, а второй на его следующего «брата». Т.е. каждый узел дерева имеет указатель на связанный список потомков, а каждый из потомков - на свой аналогичный список. Получается, что модификация бинарного дерева дает многопутевое дерево, показанное в листинге ниже.

$$
\begin{aligned}
& \text { Листинг } 8 . \\
& \text { class Tree: } \\
& \text { def__init__ ( self, kids, next=None }) \text { : } \\
& \text { self.kids = self.val = kids } \\
& \text { self.next }=\text { next }
\end{aligned}
$$

Атрибут val вводиться для получения понятного вывода при указании значения вместо ссылки на узел. Но, как и любую другую структуру еe можно изменять. Ниже приведен 
пример того, как можно обращаться с этой структурой:

>> t = Tree ( Tree ( " a ", Tree ( " b ", Tree ( " $c "$, Tree $(" d "))))$ )
>> t.kids.next.next.val

'c'

Многопутевое дерево на рисунке 3 .

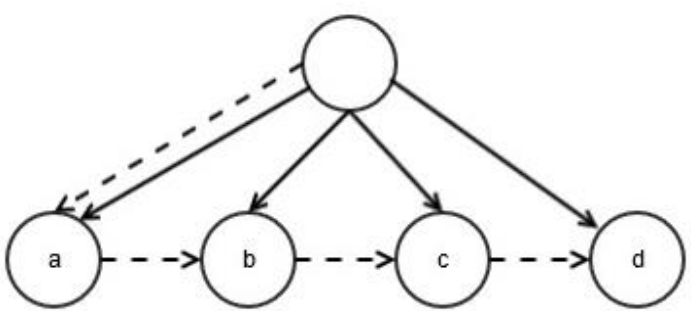

Рисунок 3 - Пример многопутевого дерева.

Пунктирными линиями на рисунке показаны атрибуты kids и next, peбра показаны сплошными линиями. В более сложном дереве вместо использования 1-го атрибута и для хранения значения узла и для ссылки на список потомков, для обеих целей могут понадобиться отдельный атрибуты.

Обычно при построении дерева будет использовать более сложных код, с четкое заданными путем.

При проектировании таких структур данных как деревья может оказаться полезным гибкий класс, позволяющий задавать набор атрибутов через конструктор. Тут лучше использовать шаблон проектирования «Набор». Есть много способов его реализации, но суть видна из следующего кода:

class Dex( dict ):

def__init_( self, *args, **kwds ): super( $\overline{D e x}$, self )._init_( $* \operatorname{args}, * * \mathrm{kwds}$

)

$$
\text { self._dict__ }=\text { self }
$$

Есть несколько полезных способов его применения. Во-первых, он позволяет создать и задать значения атрибутов, передав их как аргументы при создании объекта:

>> x = Dex(name="Drop", position="PR")

$\gg>$ x.name

'Drop'

Во-вторых, наследование от dict дает много дополнительного функционала, такого как получение всех ключей или простая проверка наличия атрибута:

$\gg>\mathrm{T}=$ Dex

$\gg>\mathrm{t}=\mathrm{T}($ left=T ( left=" a ", right=" b " ), right $=\mathrm{T}($ left $=" \mathrm{c} "))$

$\gg$ t.left

\{ 'right': 'b', 'left': 'a' \}

>> t.left.right

'b'

>> t[ 'left' ] [ 'right' ]

'b'
>> "left" in t.right

True

>> "right" in t.right

False

Использовать этот шаблон можно не только для создания деревьев. Он подойдет в любой ситуации где нужен гибкий объект, который может задавать свои атрибуты при разработке. Но лучше понять специфику собственной задачи, вместо того чтобы полагаться и использовать общие выводы. Основным фактором будет асимптотическая сложность того, какая задача будет решаться. К примеру, поиск ребра (u, v) в матрице смежности выполняется за $\Theta(1)$, а обход смежных с v вершин - за $\Theta(n)$, в то время как на списке смежности обе операции будут выполнены за $\Theta(\mathrm{d}(\mathrm{v}))$, т.е. будут зависеть от количества смежных с данной вершин. Но если в зависимости от данного типа представления асимптотическую сложность алгоритма найти невозможно, то можно провести различные эмпирические тесты. Либо же можно выбрать конкретное представление, которое будет лучше сочетаться с конкретным кодом и будет легче в поддержке.

Существует еще важный метод при использовании графов, и этот метод не касается представлений. Суть в том, что данные в большинстве задач уже имеют структуру графа или же дерева. Если зада уже имею структуру, то для них можно уже использовать соответствующие алгоритмы для графов и деревьев, не создавая особые представления. В основном это происходит, если представление графа было составлено отдельно от разрабатываемой программы. Например, если разрабатываются XML-документы или каталоги файловой системы, то древовидная структура уже создана и имеет определенные АРI. Т.е. неявно задается граф. Например, чтобы найти наиболее эффективное решение для заданной конфигурации кубика Рубика, можно определить 
состояние кубика и способы его изменения. Но даже если очевидно не описывать и не сохранять каждую конфигурацию, вероятные состояния сформируют неявный граф с ребрами операторами изменения состояния. Далее можно использовать такие алгоритмы, как $\mathrm{A}^{*}$ или двунаправленный алгоритм Дейкстры, чтобы найти кратчайший путь до состояния решения. В таких случаях функция получения примыкающих вершин N(v) будет работать «на лету», возможно, возвращая смежные вершины как коллекцию или другую форму объекта-итератора.
Граф подзадач - последний вид графов который будет рассмотрен. Большинство задач могут быть разложены на мелкие подзадачи. Эти подзадачи довольно часто имеют схожую структуру. Они формируют вершины графа подзадач, а зависимость вершин - выражаются ребрами. Хотя алгоритмы на графы редко используются на графах подзадач, такие графы отлично позволяют понять технику «разделяй и властвуй» и динамическое программирование.

\section{References:}

1. Frank Harari (2003) Graph theory - Editorial URSS, 2003.

2. Berezina (1990) Graphs and their application Moscow "Enlightenment", 1990.

3. Asanov (2010) Discrete mathematics: Graphs, matroids, algorithms - DOE, 2010.

4. Ore (1989) Graphs and their uses - World, 1989.

5. Fleisner (2002) Eulerian graphs and related topics - World, 2002.
6. Michael Bowles (2014) Machine Learning in Python: Essential Techniques for Predictive Analysis - Manning Publication, 2014.

7. Mark Lutz (2010) Python - O'Reilly Media, 2013.

8. Paul Barry (2010) Head First Python - O'Reilly Media, 2010.

9. Christopher (2001) Python \& XML - O'Reilly Media, 2001.

10. Alex Martelli (2005) Python Cookbook O'Reilly Media, 2005. 